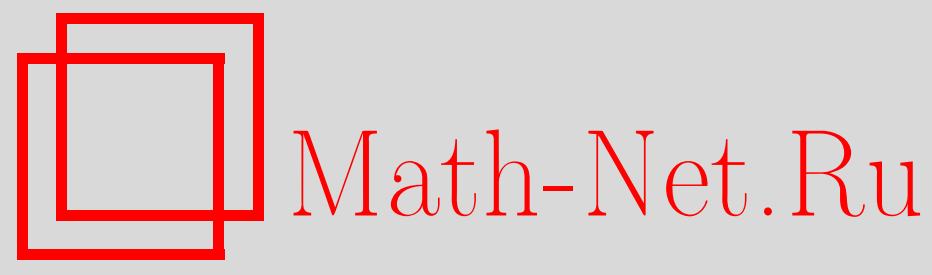

В. М. Максимов, Конечные вероятностные структуры, Дискрет. матем., 2008, том 20, выпуск 3, 19-27

DOI: https://doi.org/10.4213/dm1009

Использование Общероссийского математического портала Math-Net.Ru подразумевает, что вы прочитали и согласны с пользовательским соглашением http://www.mathnet.ru/rus/agreement

Параметры загрузки:

IP : 54.198 .187 .58

26 апреля 2023 г., $17: 58: 49$ 
УДК 519.2

\title{
Конечные вероятностные структуры
}

\author{
(c) 2008 г. В. М. Максимов
}

\begin{abstract}
Рассмотрение поля событий в теории вероятностей приводит к понятию поля событий $\mathscr{F}(B)$, состоящего из множества подмножеств некоторого множества $B$. На поле $\mathscr{F}(B)$ естественным образом определены две алгебраические структуры. Это булева алгебра $\mathscr{A}(\mathscr{F}(B))$, где в качестве операций взяты объединение, пересечение и дополнение, и решетка $L(\mathscr{F}(B))$, в которой порядок определен по включению множеств из $\mathscr{F}(B)$. В статье рассматривается еще одна алгебраическая структура на $\mathscr{F}(B)$ и ее абстрактный вариант, так называемая вероятностная структура, которая тесно связана со свойствами меры на $\mathscr{F}(B)$.
\end{abstract}

\section{1. Введение}

1. Рассмотрение поля событий в теории вероятностей естественно приводит к понятию поля событий $\mathscr{F}(B)$, состоящего из множества подмножеств некоторого множества $B$. По определению $\mathscr{F}(B)$ обладает следующим свойством: $\mathscr{F}(B)$ содержит пустое множество $\varnothing$ и $\mathscr{F}(B)$ содержит $A_{1} \cup A_{2}, A_{1} \cap A_{2}$ и $\bar{A}=B \backslash A$, если $A, A_{1}, A_{2} \in \mathscr{F}(B)$.

На поле $\mathscr{F}(B)$ естественным образом определены две алгебраические структуры. Это булева алгебра $\mathscr{A}(\mathscr{F}(B))$, где в качестве операций взяты объединение, пересечение и дополнение, и решетка $L(\mathscr{F}(B))$, в которой порядок определен по включению множеств из $\mathscr{F}(B)$.

В этой работе мы рассматриваем еще одну алгебраическую структуру на $\mathscr{F}(B)$ и ее абстрактный вариант, так называемую вероятностную структуру, которая тесно связана со свойствами меры на $\mathscr{F}(B)$.

Определение 1. Множество $\mathscr{F}(B)$ вместе с операциями сложения + , умножения · и дополнения будет называться вероятностной структурой $P(\mathscr{F}(B))$, если выполняются следующие условия.

1. Для $A_{1}, A_{2} \in \mathscr{F}(B)$ сумма $A_{1}+A_{2}$ определена тогда и и только тогда, когда $A_{1} \cap A_{2}=\varnothing$, при этом $A_{1}+A_{2}=A_{1} \cup A_{2}$.

2. Для $A_{1}, A_{2} \in \mathscr{F}(B)$ произведение $A_{1} \cdot A_{2}$ определяется как $A_{1} \cdot A_{2}=A_{1} \cap A_{2}$.

3. Для $A \in \mathscr{F}(B)$ дополнительный элемент $\bar{A}$ определяется как $\bar{A}=B \backslash A$.

Как видно, структура $P(\mathscr{F}(B))$ отличается от булевой структуры $\mathscr{A}(\mathscr{F}(B))$ только одной операцией + , которая оказывается не всегда определенной. При этом $B \in \mathscr{F}(B)$ является единичным элементом относительно умножения, а пустое множество - нулевым элементом относительно сложения. 
2. Как и в случае определения абстрактных булевых алгебр или решеток [1, 2], используя свойства вероятностной структуры $P(\mathscr{F}(B))$, можно дать абстрактное определение вероятностной структуры [3].

Определение 2. Множество $P$ с операцией + сложения, определенной для некоторых пар элементов $P$, операцией · умножения, определенной для всех пар элементов $P$, и унарной операцией дополнения $\bar{\alpha}$, определенной для всех элементов $P$, называется вероятностной структурой, если выполнены следующие условия.

1. Операция + коммутативна, то есть если $\alpha+\beta$ определено, то $\beta+\alpha$ тоже определено и $\alpha+\beta=\beta+\alpha$; ассоциативна, то есть если $\alpha+\beta$ и $(\alpha+\beta)+\gamma$ определены, то $\beta+\gamma$ и $\alpha+(\beta+\gamma)$ также определены и имеет место равенство $(\alpha+\beta)+\gamma=\alpha+(\beta+\gamma)$; и имеет нулевой элемент $\mathbf{0}, \mathbf{0}+\alpha=\alpha$ для всех $\alpha \in P$.

2. Операция (·) коммутативна, ассоциативна, и имеет единичный элемент $\mathbf{1}$ такой, что $\mathbf{1} \cdot \alpha=\alpha$ для всех $\alpha \in P$.

3. Свойство дистрибутивности справедливо в следующем смысле: если сумма $\alpha+\beta$ определена, то для всех $\gamma \in P$ сумма $\gamma \cdot \alpha+\gamma \cdot \beta$ определена и равна $\gamma \cdot(\alpha+\beta)$. Тем не менее, если для некоторых $\alpha, \beta, \gamma$ сумма $\gamma \cdot \alpha+\gamma \cdot \beta$ определена, то отсюда не следует, что определена сумма элементов $\alpha$ и $\beta$.

4. Унарная операция каждому $\alpha \in P$ ставит в соответствие элемент $\bar{\alpha} \in P$, называемый дополнительным к $\alpha$, такой, что $\alpha+\bar{\alpha}=1$. Однако, из равенства $\alpha+\beta=1$ не обязательно следует, что $\beta=\bar{\alpha}$.

5. Для всех $\alpha \neq \mathbf{0}$ сумма $\mathbf{1}+\alpha$ не определена в $P$.

Заметим, что в п. 1 определения 2 описана ситуация, когда в $P$ определена сумма трех элементов. Аналогично по индукции описывается ситуация, когда в $P$ определена сумма $n$ элементов.

3. Если множество $B$ конечно, а $\mathscr{F}(B)$ состоит из всех подмножеств множества $B$, то $\mathscr{F}(B)$ мы называем конечным булеаном (или просто булеаном) и обозначаем $\mathscr{B}$. Правда, иногда булеаном называют то, что мы называем решеткой булеана $L(\mathscr{B})$, то есть булеан $\mathscr{B}$ вместе с частичным порядком по включению множеств. Необходимость выделения булеана $\mathscr{B}$ связана с тем, что в случае конечного множества $B$ и любого поля подмножеств $\mathscr{F}(B)$ алгебраические структуры $\mathscr{A}(\mathscr{F}(B)), L(\mathscr{F}(B))$ и $P(\mathscr{F}(B))$, как легко видеть, изоморфны соответствующим структурам некоторого булеана $\mathscr{R}_{0}$.

Так же, как и в случае абстрактных булевых алгебр и решеток $[1,2]$, естественно возникает вопрос, при каких условиях абстрактная вероятностная структура $P$ является вероятностной структурой вида $P(\mathscr{F}(B))$.

Главный результат работы, который анонсирован в [3], составляет следующая теорема.

Теорема 1. Всякая конечная вероятностная структура $P$ изоморфна вероятностной структуре некоторого конечного булеана.

4. Теперь приведем некоторые соображения по поводу определения вероятностных структур.

В последние 70 лет, начиная с работы Дирака [4], появляются работы, в которых рассматриваются отрицательные, комплексные, $p$-адические и другие вероятности в контексте обоснования или объяснения физических феноменов (см., например, [5-10]). В 
ряде случаев рассмотрение неклассических вероятностей приводит к событиям с отрицательными числовыми вероятностями. Другое дело - $p$-адическая квантовая механика [11], где, по сути дела, требуется физическая интерпретация $p$-адических вероятностей. Однако, оказалось, что наиболее понятное с физической точки зрения определение $p$-адической вероятности как $p$-адического предела частоты $n_{A} / n$ события $A$ в последовательности событий не соответствует закону больших чисел для схемы Бернулли с $p$-адическими вероятностями $[12,13]$. Это обстоятельство полностью меняет взгляд на неклассические вероятности как на простое обобщение классических вероятностей из отрезка чисел $[0,1]$. Поэтому в [14] было предложено рассматривать элементы специальных алгебраических структур как абстрактные вероятности. Далее, в [15] был введен более узкий класс таких структур, названных вероятностными множествами $\mathscr{P}$, в свойствах которых заключены наиболее характерные для теории вероятностей свойства отрезка чисел $[0,1]$. Собственно, вероятностное множество $\mathscr{P}$ есть вероятностная структура, в которой, во-первых, свойство 5 заменено на более сильное свойство $5^{\prime}$, состоящее в том, что для всех $\alpha \in P, \alpha \neq \mathbf{0}$, сумма $\alpha+\ldots+\alpha$ ( $k$ раз) не определена в $P$, начиная с некоторого $k=k(\alpha)$ (см. лемму 1). Физически это отражает понятное свойство, что сколь угодно большая сумма ненулевой вероятности не может быть вероятностью. Во-вторых, добавляется так называемое условие Колмогорова: уравнение $x=x^{2}$ в $P$ имеет только два решения, $x_{1}=\mathbf{0}, x_{2}=\mathbf{1}$. Условие Колмогорова обеспечивает выполнимость закона нуль-единица для суммы независимых действительных случайных величин с $P$-значными распределениями [16].

В связи с этим заметим, что абстрактная вероятностная структура $P$ может не удовлетворять свойству $5^{\prime}$ (см. [3]) и не удовлетворять свойству Колмогорова. Например, если

$$
P=P(\mathscr{F}(B)), \quad|\mathscr{F}(B)|>2,
$$

то для всех $A \in \mathscr{F}(B)$ справедливо соотношение $A^{2}=A \cap A=A$, то есть свойство Колмогорова не выполнено.

Тем не менее, абстрактная вероятностная структура $P$ обладает минимальным набором свойств, необходимых для построения $P$-значной меры на $\mathscr{F}(B)$, обладающей естественными физическими свойствами.

Укажем на одно из таких свойств - свойство неразложимости нуля. Оно состоит в том, что множество меры нуль не может быть суммой двух непересекающихся множеств ненулевой меры, то есть из равенства $\alpha+\beta=\mathbf{0}, \alpha, \beta \in P$, должно следовать, что $\alpha=\beta=\mathbf{0}$, что доказано в разделе 2 .

В заключение заметим, что из предложения 1 и теоремы 1 вытекает следующее утверждение.

Теорема 2. Всякое конечное вероятностное множество Ф्Р может состоять только из $\mathbf{0}$ и 1, где сумма $\mathbf{1}+\mathbf{1}$ не определена и, таким образом, Я совпадает с двухэлементной вероятностной структурой.

\section{2. Общие свойства вероятностной структуры}

1. Свойство неразложимости нуля формально не входит в определение вероятностной структуры $P$, но оно является следствием из ее определения.

Лемма 1. В любой вероятностной структуре Р выполняется свойство неразложимости нуля. 
Доказательство. Пусть для $\alpha, \beta \in P$ выполнено равенство $\alpha+\beta=\mathbf{0}$. Покажем, что тогда $\alpha=\beta=\mathbf{0}$. Прибавляя к обеим частям равенства $\alpha+\beta=\mathbf{0}$ элемент $\bar{\beta}$ согласно свойству 1 определения 2, получим, что

$$
(\alpha+\beta)+\bar{\beta}=\bar{\beta}=\alpha+(\beta+\bar{\beta})=\alpha+\mathbf{1},
$$

где сумма $\alpha+\mathbf{1}$ должна быть определена в $P$. Но в силу свойства 5 определения 2 это возможно только для $\alpha=\mathbf{0}$. Следовательно $\alpha=\mathbf{0}$ и $\beta=\mathbf{0}$.

Следствие 1. Для всех $\alpha \in P$ справедливо равенство $\mathbf{0} \cdot \alpha=\mathbf{0}$.

Действительно, умножая обе части равенства $\alpha+\bar{\alpha}=\mathbf{1}$ на $\mathbf{0}$, получим, что справедливо равенство $\mathbf{0} \cdot \alpha+\mathbf{0} \cdot \bar{\alpha}=\mathbf{0} \cdot \mathbf{1}=\mathbf{0}$. Отсюда $\mathbf{0} \cdot \alpha=\mathbf{0}$ в силу леммы 1.

Легко видеть из определения 1 , что вероятностная структура $P(\mathscr{F}(B))$ булеана $\mathscr{B}$ обладает всеми свойствами вероятностного множества за исключением свойства Колмогорова, если булеан содержит более двух подмножеств.

Если $\mathscr{F}(B)$ состоит из двух множеств $B$ и $\varnothing$, то $P(\mathscr{F}(B))$ состоит только из $\mathbf{0}$ и $\mathbf{1}$ и называется элементарным $P_{0}$. Таким образом, элементарная вероятностная структура $P_{0}$ одновременно является и вероятностным множеством.

В [3] указан пример вероятностной структуры, которая в отличие от $P(\mathscr{F}(B))$ совсем не похожа на вероятностное множество, так как в ней имеется ненулевой элемент, обозначим его $\alpha$, для которого сумма $n$ слагаемых $\alpha+\ldots+\alpha=n \alpha$ определена для любого натурального $n$.

Однако имеет место следующее утверждение.

Предложение 1. Всякое вероятностное множество относительно своих операиий является и вероятностной структурой.

Доказательство. В вероятностной структуре и вероятностном множестве свойства 1-4 совпадают. Свойство Колмогорова в вероятностной структуре не требуется. Таким образом, надо только доказать, что выполняется свойство 5 определения 2, то есть, что в вероятностном множестве сумма $\mathbf{1}+\alpha$ также не определена при $\alpha \neq \mathbf{0}$.

Предположим противное, то есть предположим, что сумма $\mathbf{1}+\alpha=\gamma \in \mathscr{P}$ определена для $\alpha \neq \mathbf{0}$. Прибавляя $\bar{\gamma}$ к обеим частям равенства, получим, что

$$
(\mathbf{1}+\alpha)+\bar{\gamma}=\gamma+\bar{\gamma}=\mathbf{1}=\mathbf{1}+(\alpha+\bar{\gamma})=\mathbf{1}+\beta,
$$

где $\beta=\alpha+\bar{\gamma} \in \mathscr{P}$. Тогда к равенству $\mathbf{1}=\mathbf{1}+\beta$ можно последовательно прибавлять $\beta$, так как $(\mathbf{1}+\beta)+\beta=\mathbf{1}+\beta=\mathbf{1}=\mathbf{1}+2 \beta$. Аналогично получим, что справедливо равенство $\mathbf{1}=\mathbf{1}+n \beta$, где сумма $n \beta=\beta+\ldots+\beta$ определена при любом натуральном $n$. Но при $\beta \neq 0$ это противоречит условию $5^{\prime}$ для вероятностного множества. Следовательно, должно выполняться равенство $\alpha+\bar{\gamma}=\beta=\mathbf{0}$. Отсюда $\alpha=\mathbf{0}$ в силу свойства неразложимости нуля.

2. Дадим несколько определений.

Определение 3. Подмножество $P_{1} \subset P$, замкнутое относительно сложения, умножения и дополнения в $P$, называется вероятностной подструктурой $P$ относительно идемпотента $e \in P, e^{2}=e$, если в $P_{1}$ выполнены свойства вероятностной структуры, в которой элемент $e$ является единицей. 
Если $e=1$, то подструктуру относительно 1 будем называть собственной подструктурой $P$. Если $e-$ идемпотент $P, e \neq \mathbf{0}$, то множество $P \cdot e=\{\alpha \cdot e, \forall \alpha \in P\}$ является вероятностной подструктурой $P$ относительно $e$. Если $P_{1}-$ собственная подструктура $P$, то $P_{1} \cdot e-$ подструктура относительно идемпотента $e$.

Если взять любое подмножество $M, M \subset P$, то оно порождает собственную вероятностную подструктуру $P(M) \subseteq P$.

Определение 4. Отображение $\varphi: P_{1} \rightarrow P_{2}$ одной вероятностной структуры на другую называется гомоморфизмом, если

1. $\varphi(\alpha \cdot \beta)=\varphi(\alpha) \cdot \varphi(\beta)$;

2. если $\alpha+\beta$ определена в $P_{1}$, то $\varphi(\alpha)+\varphi(\beta)$ определено в $P_{2}$ и $\varphi(\alpha+\beta)=\varphi(\alpha)+\varphi(\beta)$;

3. $\varphi(\bar{\alpha})=\overline{\varphi(\alpha)}$.

Из определения следует, что $\varphi\left(\mathbf{1}_{1}\right)=\mathbf{1}_{2}$ и $\varphi\left(\mathbf{0}_{1}\right)=\mathbf{0}_{2}$, где $\mathbf{1}_{1}, \mathbf{0}_{1}, \mathbf{1}_{2}, \mathbf{0}_{2}-$ единица и ноль в $P_{1}$ и $P_{2}$ соответственно. Действительно,

$$
\mathbf{1}_{2}=\varphi(\alpha)+\overline{\varphi(\alpha)}=\varphi(\alpha)+\varphi(\bar{\alpha})=\varphi(\alpha+\bar{\alpha})=\varphi\left(\mathbf{1}_{1}\right) .
$$

Далее, $\varphi-$ отображение $P_{1}$ на $P_{2}$, поэтому существует элемент $\alpha \in P_{1}$ такой, что $\varphi(\alpha)=\mathbf{0}_{2}$. С другой стороны, в силу следствия 1

$$
\varphi\left(\mathbf{0}_{1}\right)=\varphi\left(\mathbf{0}_{1} \alpha\right)=\varphi\left(\mathbf{0}_{1}\right) \varphi(\alpha)=\varphi\left(\mathbf{0}_{1}\right) \mathbf{0}_{2}=\mathbf{0}_{2} .
$$

Определение 5. Если гомоморфизм $\varphi: P_{1} \rightarrow P_{2}$ взаимно однозначен, то вероятностные структуры $P_{1}$ и $P_{2}$ изоморфны.

Определение 6. Вероятностная структура на прямом произведении $P_{1} \times P_{2}$ вероятностных структур $P_{1}$ и $P_{2}$ определяется операциями покомпонентного умножения и сложения, если сложение определено в каждой компоненте.

Очевидно, что $P_{1} \times P_{2}$ будет вероятностной структурой, если положить $\mathbf{0}=\left(\mathbf{0}_{1}, \mathbf{0}_{2}\right)$, $\mathbf{1}=\left(\mathbf{1}_{1}, \mathbf{1}_{2}\right)$ и $\overline{\left(\alpha_{1}, \alpha_{2}\right)}=\left(\bar{\alpha}_{1}, \bar{\alpha}_{2}\right)$.

Аналогично определяется вероятностная структура на прямом произведении с произвольным числом сомножителей.

3. Пусть $\mathscr{B}_{1}$ и $\mathscr{B}_{2}$ - два булеана, заданные на непересекающихся множествах $B_{1}$ и $B_{2}$. Тогда прямое произведение $\mathscr{B}_{1} \times \mathscr{B}_{2}$ есть булеан, заданный на $B_{1} \cup B_{2}$ подмножествами $\left\{A_{1} \cup A_{2}\right\}$, где $A_{1} \in \mathscr{B}_{1}, A_{2} \in \mathscr{B}_{2}$. Обозначим $P(\mathscr{B})$ вероятностную структуру булеана $\mathscr{B}$. Тогда очевидно следующее утверждение.

Предложение 2. Справедливо равенство $P\left(\mathscr{B}_{1} \times \mathscr{B}_{2}\right)=P\left(\mathscr{B}_{1}\right) \times P\left(\mathscr{B}_{2}\right)$.

4. Рассмотрим теперь формальную вероятностную структуру $B_{s}$, состоящую из $2^{s}$ элементов 0, 1, $e_{1}, e_{2}, \ldots, e_{s}$, и всех формальных выражений вида $\left\{e_{i_{1}}+\ldots+e_{i_{k}}\right\}$, $1 \leqslant i_{1}<i_{2}<\ldots<i_{k} \leqslant s$, причем $e_{1}+e_{2}+\ldots+e_{s}=\mathbf{1}$.

Введем операции умножения $e_{i} \cdot e_{i}=e_{i}, e_{i} \cdot e_{j}=\mathbf{0}$ для $i=1, \ldots, s, i \neq j$. Сумма $\left\{e_{i_{1}}+\ldots+e_{i_{k}}\right\}+\left\{e_{j_{1}}+\ldots+e_{j_{m}}\right\}$ не определена, если совпадает хотя бы один индекс в первом и во втором выражении. Если все индексы различны, то сумма определена и равна $\left\{e_{i_{1}}+\ldots+e_{i_{k}}+e_{j_{1}}+\ldots+e_{j_{m}}\right\}$. Операции умножения выражений доопределяются 
по дистрибутивности. Дополнение $\overline{\left\{e_{i_{1}}+\ldots+e_{i_{k}}\right\}}$, очевидно, равно $\left\{e_{j_{1}}+\ldots+e_{j_{s-k}}\right\}$, где индексы $j_{1}, \ldots, j_{s-k}$ отличны от $i_{1}, \ldots, i_{k}$. Легко видеть, что с такими операциями множество $B_{s}$ является вероятностной структурой.

Отсюда очевидным образом вытекает следующее утверждение.

Предложение 3. Вероятностная структура конечного булеана, содержашего $2^{s}$ подмножеств, изоморфна вероятностной структуре $B_{s}$.

Доказательство. Действительно, рассмотрим булеан, образованный множеством натуральных чисел $\{1,2, \ldots, s\}$. Сопоставим каждому подмножеству $\left\{i_{1}, i_{2}, \ldots, i_{k}\right\}$, где $1 \leqslant i_{1}<i_{2}<\ldots<i_{k} \leqslant s$, выражение $\left\{e_{i_{1}}+\ldots+e_{i_{k}}\right\}$, а пустому множеству сопоставим $\mathbf{0}$.

Легко также видеть, что вероятностная структура $B_{s}$ изоморфна прямому произведению $s$ элементарных вероятностных структур $P_{0}$. Изоморфизм осуществляется соответствием

$$
\begin{gathered}
\mathbf{0} \leftrightarrow(\mathbf{0}, \mathbf{0}, \ldots, \mathbf{0}), \quad \mathbf{1} \leftrightarrow(\mathbf{1}, \mathbf{1}, \ldots, \mathbf{1}), \\
e_{i_{1}}+\ldots+e_{i_{k}} \leftrightarrow\left(\delta_{1}, \ldots, \delta_{s}\right),
\end{gathered}
$$

где $\delta_{i_{1}}=\delta_{i_{2}}=\ldots=\delta_{i_{k}}=\mathbf{1}$, а остальные $\delta_{j}=\mathbf{0}$.

Учитывая предложение 3, получаем следующее утверждение.

Предложение 4. Вероятностная структура булеана, содержащего $2^{s}$ подмножеств, изоморфна прямому произведению $s$ элементарных вероятностных структур $P_{0}$.

\section{3. Несколько свойств вероятностной структуры $P$, вытекающих из неопределяемости суммы $1+\alpha$ или из конечности $P$}

\section{1. Свойства $P$, связанные с неопределяемостью $1+\alpha$.}

Лемма 2. Если для некоторых $\alpha, \beta \in P$ справедливо равенство $\alpha+\beta=\alpha$, то $\beta=\mathbf{0 .}$

Доказательство. Прибавляя к обеим частям равенства $\alpha+\beta=\alpha$ элемент $\bar{\alpha}$, получим, что

$$
(\alpha+\beta)+\bar{\alpha}=\alpha+\bar{\alpha}=\mathbf{1}=\beta+(\alpha+\bar{\alpha})=\beta+\mathbf{1}
$$

это противоречит условию 5 определения 2 , если $\beta \neq 0$.

Лемма 3. Пусть е - ненулевой идемпотент в $P$. Тогда сумма $е+e$ не определена в $P$.

Доказательство. Допустим противное, то есть, что сумма $e+e=\beta$ определена в $P$. Тогда, прибавляя $\bar{\beta}$ к обеим частям равенства, получим, что $(e+e)+\bar{\beta}=\beta+\bar{\beta}=\mathbf{1}=$ $e+(e+\bar{\beta})$. Умножая равенство $1=e+(e+\bar{\beta})$ на $e$ и учитывая, что $e^{2}=e$, получим, что $e=e+(e+\bar{\beta}) e$. В силу леммы $2,(e+\bar{\beta}) e=e+\bar{\beta} e=\mathbf{0}$. Отсюда, в силу неразложимости нуля, получим, что $e=\mathbf{0}$, что противоречит нашему предположению.

Лемма 4. В любой вероятностной структуре $P$ дополнительный элемент $\bar{e}$ для идемпотента е $\in P$ является идемпотентом ортогональным ке, то есть, е $\bar{e}=\mathbf{0}$. 
Доказательство. Умножая равенство $e+\bar{e}=1$ на $e$, получим, что $e^{2}+\bar{e} e=e=e+\bar{e} e$. Отсюда, в силу леммы $2, \bar{e} e=\mathbf{0}$. Умножая теперь равенство $e+\bar{e}=\mathbf{1}$ на $\bar{e}$ и учитывая, что $\bar{e} e=\mathbf{0}$, получим равенство $\bar{e}^{2}=\bar{e}$.

Лемма 5. В любой вероятностной структуре $P$ элемент $\bar{e}$, дополнительный для идемпотента е $\in P$, является единственным решением уравнения $e+x=1$.

Доказательство. Пусть имеется еще элемент $\bar{e}_{1} \in P$, для которого $e+\bar{e}_{1}=\mathbf{1}$. Тогда, в силу леммы 4 , элементы $\bar{e}_{1}$ и $\bar{e}$ ортогональны $e$. Поэтому, умножая равенство $e+\bar{e}=1$ на $\bar{e}_{1}$, а равенство $e+\bar{e}_{1}=\mathbf{1}$ на $\bar{e}$, получаем, что $\bar{e}_{1}=\bar{e}_{1} \bar{e}, \bar{e}=\bar{e}_{1} \bar{e}$, то есть $\bar{e}_{1}=\bar{e}$.

\section{2. Свойства, связанные с конечностью $P$.}

Лемма 6. В конечной вероятностной структуре $P$ для всех $\alpha \neq \mathbf{0}$ сумма $\alpha+\ldots+\alpha$ ( $k$ раз) не определена, начиная с некоторого $k, k=k(\alpha)$.

Доказательство. Допустим, что сумма $\alpha+\ldots+\alpha=m \alpha$ определена при любом числе слагаемых $m$. При этом $m \alpha+k \alpha=(m+k) \alpha$. Так как предполагается, что $P-$ конечное множество, в последовательности $\{m \alpha\}$ может быть только конечное число различных членов. При некоторых натуральных $k$ и $m$ поэтому $(m+k) \alpha=m \alpha=m \alpha+k \alpha$. В силу леммы 2 получаем, что $k \alpha=\mathbf{0}$ или $k \alpha=(k-1) \alpha+\alpha=\mathbf{0}$. В силу следствия 1 о неразложимости нуля, отсюда следует, что $\alpha=\mathbf{0 .}$

Таким образом, конечная вероятностная структура удовлетворяет всем свойствам вероятностного множества, кроме, может быть, условия Колмогорова.

Следующее утверждение справедливо для вероятностного множества и доказано в [15]. Однако оно справедливо и для произвольной конечной вероятностной структуры.

Предложение 5. Пусть $P$ - произвольная конечная вероятностная структура. Если для некоторого $\alpha \in P$ справедливо равенство $\alpha^{2}=\mathbf{0}$, то $\alpha=\mathbf{0}$.

Доказательство. Умножая равенство $\alpha+\bar{\alpha}=1$ на $\alpha$, получим, что $\alpha^{2}+\bar{\alpha} \alpha=\alpha$. По условию $\alpha^{2}=\mathbf{0}$, поэтому справедливо равенство $\bar{\alpha} \alpha=\alpha$. С другой стороны,

$$
\mathbf{1}=(\alpha+\bar{\alpha})=(\alpha+\bar{\alpha})^{2}=\alpha^{2}+2 \alpha \bar{\alpha}+\bar{\alpha}^{2} .
$$

Отсюда, учитывая, что $\alpha \bar{\alpha}=\alpha$ и $\alpha^{2}=\mathbf{0}$, получаем основное равенство $2 \alpha+\bar{\alpha}^{2}=\mathbf{1}$. Умножая это равенство на $\bar{\alpha}$ и заменяя $\bar{\alpha} \alpha$ на $\alpha$, получим, что $2 \alpha+\bar{\alpha}^{3}=\bar{\alpha}$. Наконец, прибавляя к обеим частям по $\alpha$, получим равенство $3 \alpha+\bar{\alpha}^{3}=\mathbf{1}$.

Продолжая аналогичным образом, мы придем к равенству $n \cdot \alpha+\bar{\alpha}^{n}=\mathbf{1}$, справедливому при любом натуральном $n$. Это значит, что сумма $n \cdot \alpha=\alpha+\ldots+\alpha$ определена при любом $n$. В силу леммы 6 , для конечного $P$ это возможно только при $\alpha=\mathbf{0}$.

\section{4. Доказательство теоремы 1}

1. Имеет место следующее утверждение.

Предложение 6. В конечной вероятностной структуре $P$ можно определить такое натуральное $k(P)$, что в $P$ существуют $k(P)$ ненулевых элементов $y_{1}, y_{2}, \ldots, y_{k(P)}$, не обязательно различных, таких, что их сумма определена и $y_{1}+y_{2}+\ldots+y_{k(P)}=1$. При этом, для любых $k(P)+1$ ненулевых элементов $P$ их сумма не определена в $P$. 
Доказательство. Допустим, что в $P$ существуют ненулевые наборы таких элементов $\left\{y_{1}^{(i)}, y_{2}^{(i)}, \ldots, y_{n_{i}}^{(i)}\right\}, i=1,2, \ldots$, что $n_{i} \rightarrow \infty$ при $i \rightarrow \infty$ и $y_{1}^{(i)}+\ldots+y_{n_{i}}^{(i)}=\mathbf{1}$. Тогда, в силу конечности множества $P$, среди данного набора множеств $\left\{y_{1}^{(i)}, \ldots, y_{n_{i}}^{(i)}\right\}$ найдется бесконечное подмножество наборов $\left\{y_{1}^{(j)}, \ldots, y_{n_{j}}^{(j)}\right\}$, в которых некоторый элемент $y \in P$, $y \neq \mathbf{0}$, встречается $r(j)$ раз и $r(j) \rightarrow \infty$ при $j \rightarrow \infty$. Следовательно, сумма $y+\ldots+y$ должна быть определена для любого числа слагаемых, что противоречит лемме 6. Поэтому существует такое наибольшее натуральное $k(P)$, для которого существует набор из $k(P)$ ненулевых элементов $P$ в сумме дающих 1.

2. Будем говорить, что всякий набор ненулевых элементов $y_{1}, y_{2}, \ldots, y_{m}$, для которого $y_{1}+\ldots+y_{m}=1$, образует разложение 1 . В силу предложения $6, m \leqslant k(P)$. С другой стороны, $y+\bar{y}=\mathbf{1}$, поэтому $k(P) \geqslant 2$.

Предложение 7. Если в конечной вероятностной структуре $P$ элементы $y_{1}, \ldots, y_{k}(P)$ из $P$ образуют разложение 1, то они являются ортогональными идемпотентами.

Доказательство. Возведем равенство $y_{1}+\ldots+y_{k(P)}=1$ в квадрат. Тогда

$$
\sum_{i=1}^{k(P)} y_{i}^{2}+\sum_{i \neq j} y_{i} y_{j}=\mathbf{1} \text {. }
$$

Так как все $y_{i} \neq \mathbf{0}$, в силу предложения 5 все $y_{i}^{2} \neq \mathbf{0}$. Поэтому в силу определения числа $k(P)$ в предложении 6 остальные члены разложения 1 должны быть равны $\mathbf{0}$, то есть в последнем разложении 1 все $y_{i} \cdot y_{j}=\mathbf{0}$ при $i \neq j$. Отсюда и из предложения 5 следует, что среди $y_{1}, \ldots, y_{k(P)}$ не может быть равных элементов. Таким образом, в разложении $y_{1}+\ldots+y_{k(P)}=\mathbf{1}$ все $y_{i}$ различны. Поэтому, умножая это разложение $\mathbf{1}$ последовательно на $y_{1}, y_{2}, \ldots, y_{k(P)}$ и учитывая, что $y_{i} y_{j}=\mathbf{0}$ при $i \neq j$, получаем, что $y_{i}^{2}=y_{i}$.

3. Обозначим эти ненулевые различные идемпотенты через $e_{1}, e_{2}, \ldots, e_{s}$, где $s=k(P)$, $e_{1}+\ldots+e_{s}=\mathbf{1}, e_{i} \cdot e_{j}=\mathbf{0}, i \neq j$. Равенство $e_{1}+\ldots+e_{s}=\mathbf{1}$ означает, что в $P$ определены суммы $e_{i_{1}}+\ldots+e_{i_{k}}, 1 \leqslant i_{i}<i_{2}<\ldots<i_{k} \leqslant s$.

Очевидно, что множество, состоящее из $\mathbf{0}, 1$ и всех элементов $e_{i_{1}}+\ldots+e_{i_{k}}$, $1 \leqslant i_{i}<i_{2}<\ldots<i_{k} \leqslant s$, является вероятностной структурой $B_{s}$, и эта структура является собственной вероятностной подструктурой в $P$.

Предложение 8. Собственная вероятностная подструктура $B_{s}$ в P совпадает c P.

Доказательство. Достаточно показать, что $P \subseteq B_{s}$. Пусть $y-$ произвольный ненулевой элемент $P$. Так как $e_{i}=e_{i}(y+\bar{y})=e_{i} y+e_{i} \bar{y}$, в разложении единицы $e_{1}+\ldots+e_{s}=\mathbf{1}$ элемент $e_{i}$ можно заменить суммой $e_{i} y+e_{i} \bar{y}$. Поэтому

$$
e_{1}+\ldots+e_{i-1}+e_{i} y+e_{i} \bar{y}+e_{i+1}+\ldots+e_{s}=\mathbf{1} \text {. }
$$

Разложение единицы не может содержать более $s$ элементов, поэтому либо $e_{i} y=\mathbf{0}$, $e_{i} \bar{y} \neq \mathbf{0}$, либо $e_{i} y \neq \mathbf{0}$, но $e_{i} \bar{y}=\mathbf{0}$.

Пусть $i_{1}<\ldots<i_{m}-$ все те номера, для которых $e_{i_{1}} y \neq \mathbf{0}, \ldots, e_{i_{m}} y \neq \mathbf{0}$, но $e_{i_{1}} \bar{y}=\ldots=e_{i_{m}} \bar{y}=\mathbf{0}$.

Поэтому в разложении единицы, заменяя каждое $e_{i}$ на сумму $e_{i} y+e_{i} \bar{y}$, получаем, что

$$
e_{i_{1}} y+\ldots+e_{i_{m}} y+\sum_{j \notin\left\{i_{1}, \ldots, i_{m}\right\}} e_{j} \bar{y}=\mathbf{1} .
$$


Умножая это равенство последовательно на $e_{1}, e_{2}, \ldots, e_{s}$ и учитывая ортогональность $e_{i}$, получаем, что $e_{i_{1}} y=e_{i_{1}}, \ldots, e_{i_{m}} y=e_{i_{m}}$.

Снова возвратимся к разложению единицы $e_{1}+\ldots+e_{s}=1$. Умножая это равенство на $y$ и учитывая, что $e_{i_{1}} y=e_{i_{1}}, \ldots, e_{i_{m}} y=e_{i_{m}}$, но $e_{j} y=\mathbf{0}$, для $j \notin\left\{i_{1}, \ldots, i_{m}\right\}$, получаем, что $y=e_{i_{1}}+\ldots+e_{i_{m}} \in P$.

Заметим теперь, что из предложений 4 и 8 следует, что всякая конечная вероятностная структура изоморфна вероятностной структуре конечного булеана, что завершает доказательство теоремы 1.

Пользуясь случаем, приношу благодарность О. В. Вискову, А. Ю. Хренникову, Э. Л. Пресману, А. М. Степину, Б. С. Стечкину за внимание к работе и полезные замечания.

\section{Список литературы}

1. Сикорский Р., Булевы алгебры. Мир, Москва, 1969.

2. Биркгоф Г., Теория решеток. Наука, Москва, 1984.

3. Максимов В. М., Вероятностные структуры и вероятностные множества. Успехи матем. наук (2007) 62, №6 (378), 181-182.

4. Dirac P. A. M., On the analogy between classical and quantum mechanics. Rev. Modern Phys. (1945) 17, 195-199.

5. Feynman R. P., Negative probability. Quantum implications. Routledge and Kegan Paul, London, 1987.

6. Хренников А. Ю., p-адическая вероятность и статистика. Докл. РАН (1992) 322, 1075-1079.

7. Khrennikov A. Yu., p-adic valued distributions and their applications to the mathematical physics. Kluwer, Dordrecht, 1994.

8. Хренников А. Ю., О расширении частотного подхода Р. фон Мизеса и аксиоматического подхода А. Н. Колмогорова на $p$-адическую теорию вероятностей. Теория вероятностей и ее применения (1995) 40, №2, 458-463.

9. Хренников А. Ю., Неархимедов анализ и его применения. Наука, Москва, 2003.

10. Khrennikov A. Yu., Generalized probabilities taking values in non-Archimedean fields and topological groups. Russ. J. Math. Phys. (2007) 14, №2, 142-159.

11. Vladimirov V. S., Volovich I. V., Zelenov E. I., p-adic analysis and mathematical physics. World Scientific, Singapore, 1993.

12. Khrennikov A. Yu., $p$-adic quantum mechanics with $p$-adic valued functions. J. Math. Phys. (1991) 32, №4, 932-937.

13. Хренников А. Ю., Теорема Бернулли для вероятностей, принимающих $р$-адические значения. Докл. РАН (1997) 351, №4, 461-464.

14. Maximov V. M., Abstract models of probabilities, foundations of probability and physics. In: Quantum Probab. White Noise Anal. World Scientific, River Edge, 2007, pp. 257-273.

15. Maximov V. M., The set of abstract probabilities. In: Foundations of Probability and Physics, ATP Conf. Proc.. Amer. Inst. Phys., Melville, 2007, pp. 353-361. 